\title{
Resultados auditivos del tratamiento quirúrgico de la otoesclerosis en el Departamento de Otorrinolaringología de la Pontificia Universidad Católica de Chile
}

\author{
Auditory results of surgical treatment of otosclerosis in the \\ Department of Otolaryngology of the Pontificia Universidad Católica de Chile
}

Karina Lira $\mathbf{R}^{1}$, Andrés Rosenbaum $\mathrm{F}^{1}$, Francisco García-Huidobro $\mathbf{N}^{1}$, Jorge Astudillo $\mathrm{S}^{1}$, Bárbara Huidobro D' ${ }^{1}$, Jorge Caro L'1 , José San Martín P1.

\begin{abstract}
RESUMEN
Introducción: La otoesclerosis representa la causa de hipoacusia de conducción más común en adultos jóvenes. Las opciones de tratamiento incluyen amplificación mediante audífonos o resolución quirúrgica a través de cirugías como estapedectomía total o parcial, estapedostomía o implante coclear.

objetivo: Describir los resultados del tratamiento quirúrgico de la otoesclerosis en el Departamento de Otorrinolaringología del Hospital Clínico de la Pontificia Universidad Católica de Chile entre los años 1999 y 2018.

Material y método: Se realizó un estudio de cohorte no concurrente con revisión de fichas clínicas y protocolos operatorios de pacientes atendidos en nuestro centro. Se registraron y analizaron las características biodemográficas de los pacientes, técnicas quirúrgicas, resultados auditivos, complicaciones, necesidad de reintervención y uso de audífono.

Resultados: De un total de 78 pacientes sometidos a estapedectomía parcial (platinectomía parcial) o estapedostomía se incluyeron finalmente 37 quienes tenían datos clínicos completos, lo que corresponde al 47,4\% del total. En ambos tipos de cirugía el promedio de gap óseo-aéreo varió de 30,8 dB en el preoperatorio a 13,9 dB en el posoperatorio lo cual es estadísticamente significativo. El promedio de variación de la vía aérea, éste fue mayor en el grupo de pacientes operados de estapedostomía, sin existir diferencias estadísticamente significativas al realizar test no paramétricos.

Conclusión: Las características demográficas y los resultados auditivos obtenidos en nuestro centro son comparables con los descritos en la literatura. No existen diferencias significativas en cuanto al tipo de cirugía y la frecuencia y tipo de complicaciones, aunque se observan mejores resultados auditivos en los pacientes operados de estapedostomía.
\end{abstract}

Palabras clave: Estapedectomía, estapedostomía, éxito auditivo.

\footnotetext{
1 Departamento de Otorrinolaringología, Pontificia Universidad Católica de Chile, Santiago, Chile.

Los autores declaran no tener conflictos de interés.
}

Recibido el 7 de abril, 2019. Aceptado el 30 de julio, 2019. 


\section{ABSTRACT}

Introduction: Otosclerosis represents the most common cause of hearing loss in young adults. Treatment options include amplification with hearing aids or surgical resolution with surgeries such as total and partial stapedectomy, stapedostomy or cochlear implant.

Aim: To describe the results of the surgical treatment of otosclerosis in the Otorhinolaryngology Department of the Clinical Hospital of the Pontificia Universidad Católica de Chile between the years 1999 and 2018

Material and method: A non-concurrent cohort study was performed with review of clinical files and operative protocols of patients seen in our center. The biodemographic characteristics of the patients, surgical techniques, auditory results, complications, need for reoperation and use of hearing aid were recorded and analyzed.

Results: Of a total of 78 patients undergoing partial stapedectomy or stapedostomy, 37 were finally included, who had complete clinical data, which corresponds to $47.4 \%$ of the total. In both types of surgery, the average bone-air gap varied from $30.8 \mathrm{~dB}$ in the preoperative to $13.9 \mathrm{~dB}$ in the postoperative period, which is statistically significant. Regarding the average of variation of the airway, this was higher in the group of patients operated by stapedostomy without statistically significant differences when performing nonparametric tests.

Conclusion: The demographic characteristics and the auditory results obtained in our center are comparable with those described in the literature. There are no significant differences in the type of surgery and the frequency and type of complications, although better hearing results are observed in patients operated by stapedostomy.

Key words: Stapedectomy, stapedostomy, auditory success.

\section{INTRODUCCIÓN}

La otoesclerosis en una enfermedad hereditaria autosómica dominante de baja penetrancia que afecta el hueso temporal ${ }^{1,2}$. Esta patología representa la causa de hipoacusia de conducción más común en adultos jóvenes y afecta al 0,3\% de la población. Sin embargo, la incidencia histopatológica de hueso temporal se ha descrito de hasta $13 \%{ }^{3}$. Es más frecuente en mujeres con una relación de dos es a uno $0^{1,4}$ y se presenta la mayoría de las veces con una hipoacusia lentamente progresiva, bilateral en el $80 \%$ de los casos y asimétrica ${ }^{5,6}$. En estos pacientes existe enfermedad de la cápsula ótica y dependiendo del sitio de la cápsula que se afecte, será el tipo de hipoacusia. Si es en torno a la platina será conductiva. En algunos pacientes con enfermedad avanzada, las lesiones se pueden extender hacia el laberinto óseo del oído interno afectando la cóclea y resultando en hipoacusia mixta o sensorioneural ${ }^{5}$.

El diagnóstico está basado generalmente en la historia clínica de hipoacusia progresiva en un pa- ciente con tímpano sano asociado a hallazgos en la audiometría ${ }^{6}$ tales como hipoacusia de conducción o mixta si hay compromiso coclear. Puede presentarse el signo «escotadura de Carhart» que consiste en una baja en la vía ósea en las frecuencias de los $2 \mathrm{kHz}$ acercándose a la vía aérea, hallazgo descrito por numerosos autores ${ }^{2,5,7,11}$. La impedanciometría puede mostrar curva A o As y reflejos negativos, efecto on-offy reflejos invertidos descritos por Jerger y cols ${ }^{7}$. La tomografía computarizada de oídos es un apoyo para el diagnóstico diferencial y la planificación de la cirugía ${ }^{1,7}$.

Las opciones de tratamiento incluyen amplificación mediante audífonos o resolución quirúrgica a través de cirugías como estapedectomía total 0 parcial, estapedostomía 0 implante coclear ${ }^{4,6}$, siendo generalmente considerado como más efectivo el tratamiento quirúrgico $0^{4,6,8}$. La estapedectomía y estapedostomía son procedimientos bien establecidos en el tratamiento de la otoesclerosis, generando niveles satisfactorios de audición, definidos como cierre de gap óseo-aéreo a valores menores 
o iguales a $10020 \mathrm{~dB}$ dependiendo del criterio de éxito utilizado por diferentes autores 3 ,4,9-12.

\section{OBJETIVO}

Describir los resultados del tratamiento quirúrgico de la otoesclerosis en el Departamento de Otorrinolaringología del Hospital Clínico de la Pontificia Universidad Católica de Chile entre los años 1999 y 2018.

\section{MATERIAL Y MÉTODO}

Se realizó un estudio de cohorte no concurrente con revisión de fichas clínicas y protocolos operatorios de pacientes atendidos en el Departamento de Otorrinolaringología del Hospital Clínico de la Pontificia Universidad Católica de Chile entre los años 1999 y 2018. Fueron excluidos los pacientes sin control audiométrico posoperatorio, al igual que los pacientes cuyas fichas poseían datos incompletos. Se registraron y analizaron las características biodemográficas de los pacientes, técnicas quirúrgicas, resultados auditivos, complicaciones, necesidad de reintervención y uso de audífono.

En el análisis estadístico se utilizó el programa STATA/MP ${ }^{\circledR}$ versión 13,0 . Se realizó estadística descriptiva, prueba $t$ student muestras pareadas, al evaluar variaciones dentro de cada grupo, $t$ student muestras independientes y ANOVA one way, al comparar ambos grupos, lo cual fue confirmado con las pruebas no paramétricas rangos signados de Wilcoxon, test de Mann-Whitney y KruskalWallis. Las variables categóricas se analizaron con test Chi-cuadrado. Se consideró un valor de $p$ significativo menor 0,05 .

\section{RESULTADOS}

De un total de 78 pacientes sometidos a manejo quirúrgico de otoesclerosis se incluyeron finalmente 37 quienes tenían datos clínicos completos, lo que corresponde al $47,4 \%$ del total. El seguimiento promedio fue de 2,6 años (rango de 0,25-16 años). La edad promedio fue de 45 años (rango 27 a 68 años), predominando el sexo femenino en $67,5 \%$.
Respecto al estado de avance de la enfermedad en el $83,7 \%$ de los pacientes el compromiso fue bilateral, encontrándose en 20 pacientes hipoacusia de conducción y en 17 pacientes hipoacusia mixta. En cuanto al control imagenológico el $64,9 \%$ de los pacientes contaban con tomografía computarizada de oídos, encontrándose 6 pacientes con otoesclerosis fenestral bilateral, 5 pacientes con otoesclerosis fenestral y retrofenestral bilateral, 5 pacientes con tomografía normal, 4 pacientes con otoesclerosis fenestral unilateral, 2 pacientes con cambios posquirúrgicos con prótesis in situ, un paciente con compromiso fenestral y retrofenestral unilateral y un paciente con compromiso sólo retrofenestral (Tabla 1).

En los 37 pacientes se realizó manejo quirúrgico, en 21 se hizo estapedostomía y en 16 estapedectomía parcial. En el $51,3 \%$ de los pacientes el oído operado fue el derecho y en $46 \%$ el izquierdo, existiendo 2,7\% no consignado en la ficha clínica. Solo 1 paciente fue operado bilateralmente $(2,7 \%)$. Con respecto a las complicaciones presentadas durante el seguimiento de los pacientes posterior a la cirugía, la más frecuente fue vértigo que se presentó en 15 pacientes (40,5\%), seguido de disgeusia $(24,3 \%)$, tinnitus $(16,2 \%)$, desplazamiento de prótesis $(8,1 \%)$, hipoacusia $(2,7 \%)$ e intrusión de platina $(2,7 \%)$. Dos pacientes fueron reintervenidos por desplazamiento de la prótesis (Tabla 1).

\section{Resultados audiológicos}

El promedio de gap óseo-aéreo varió de 30,8 dB en el preoperatorio a $13,9 \mathrm{~dB}$ en el posoperatorio independiente del tipo de cirugía, lo que implica una disminución de 16,9 dB que es estadísticamente significativa $(p<0,001)$ (Tabla 2). Al analizar por subgrupos, el promedio de variación del gap óseoaéreo es mayor en los pacientes en los cuales se realizó estapedostomía (22,34 dB) en comparación con los que se realizó estapedectomía parcial $(10,47 \mathrm{~dB})$, existiendo diferencias estadísticamente significativas entre ambos grupos $(p<0,05)$ (Tabla 3$)$.

Por otro lado, el PTP aéreo promedio varió de $56,58 \mathrm{~dB}$ preoperatorio a $31,42 \mathrm{~dB}$ posoperatorio independiente del tipo de cirugía, resultado una disminución de 25,2 dB lo cual que es estadísticamente significativo $(p<0,001)$. Al analizar por subgrupos, se observó que, si bien 
Tabla 1. Características clínicas y demográficas

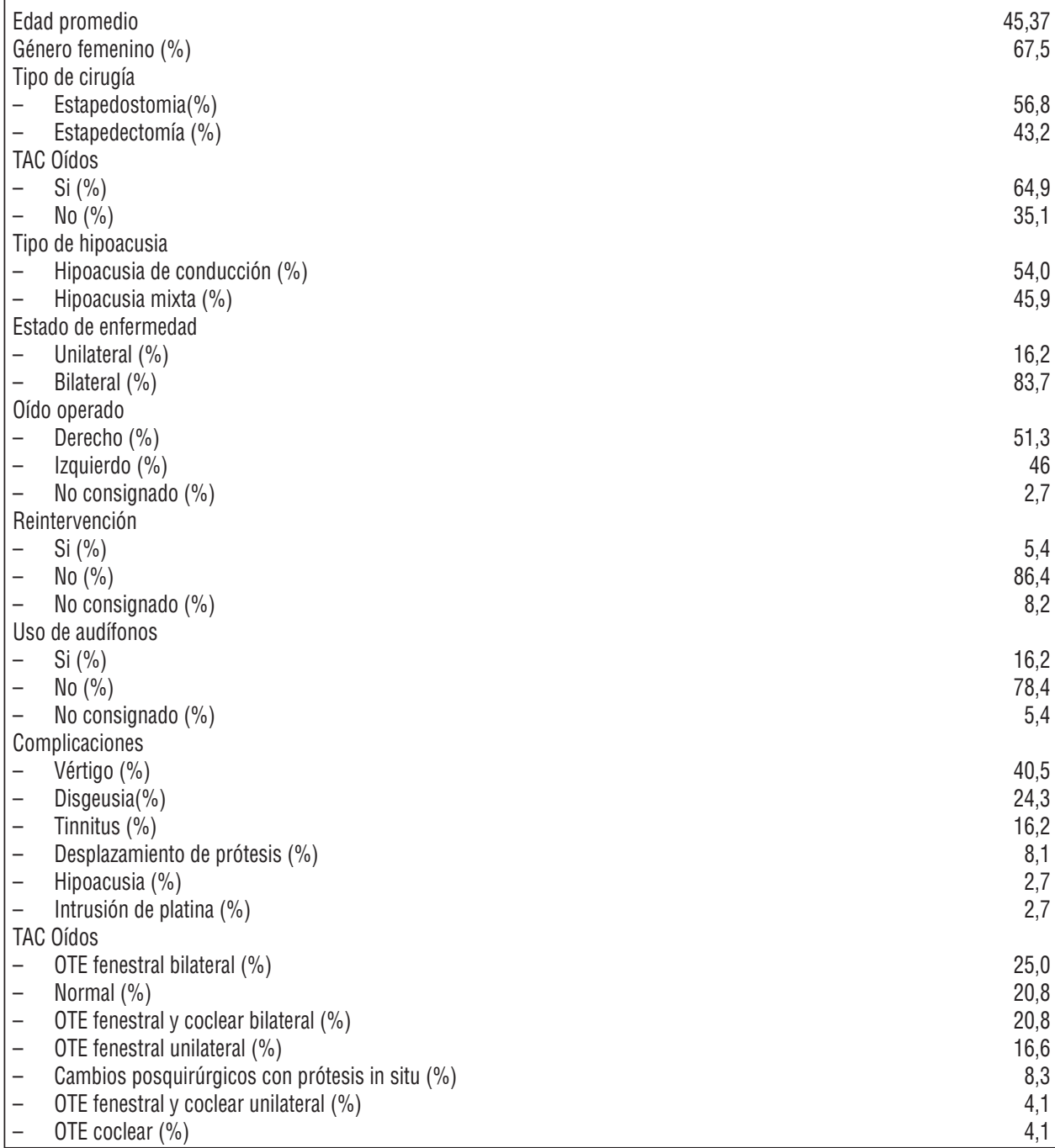

OTE: Otoesclerosis

Tabla 2. Diferencia entre GAP óseo-aéreo (dB) preoperatorio y GAP óseo-aéreo posoperatorio

\begin{tabular}{|lcrr|}
\hline Variable & Media & Desviación estándar & IC (95\%) \\
\hline GAP Preop & 30,89 & 10,87 & $27,26-34,52$ \\
GAP posop & 13,97 & 12,42 & $9,83-18,12$ \\
Diferencia & 16,91 & 16,55 & $11,39-22,43$ \\
\hline
\end{tabular}

la disminución promedio del PTP aéreo fue de mayor magnitud en el grupo al que se realizó estapedostomía (30,6 dB) en comparación con el grupo de estapedectomía parcial $(17,3 \mathrm{~dB})$, esta diferencia no es estadísticamente significativa ( $p$ $=0,29$ ) (Tabla 4).

En cuanto a las complicaciones, se observa que no existe asociación entre el tipo de cirugía y 
Tabla 3. Variación de GAP óseo-aéreo (dB) de acuerdo a tipo de cirugía

\begin{tabular}{|lcccc|}
\hline CIRUGIA & GAP preop & GAP posop & Disminución & Desviación estándar \\
\hline Estapedectomía & 28,82 & 18,35 & 10,47 & 18,45 \\
Estapedostomía & 31,69 & 9,36 & 22,33 & 13,71 \\
\hline
\end{tabular}

Tabla 4. Media de variación de delta de vía aérea (dB) de acuerdo a tipo de cirugía

\begin{tabular}{|ll|}
\hline Cirugía & Media \\
\hline Estapedectomía & 17,34 \\
Estapedostomía & 30,69 \\
Total & 25,19 \\
\hline
\end{tabular}

una mayor frecuencia de complicaciones. Si bien la frecuencia de tinnitus y disgeusia fue mayor en los pacientes en los que se realizó estapedostomía ( 4 versus 2 casos y 8 versus 2 casos, respectivamente), no existe una asociación estadísticamente significativa ( $p=0,58$ y 0,067 , respectivamente). Por otro lado, si bien la frecuencia de vértigo es mayor en el grupo de estapedectomía parcial (8 vs 7) no existe una asociación estadísticamente significativa entre estas variables $(p=0,53)$ (Tabla 1). En el caso de uso de audífonos, la frecuencia global de su uso posterior a la intervención quirúrgica fue de $16,2 \%$, siendo mayor en el grupo de pacientes en los que se realizó estapedectomía parcial, lo cual no es estadísticamente significativo $(p=0,255)$.

\section{DISCUSIÓN}

Existen opciones de tratamiento para la otoesclerosis que incluyen amplificación mediante audífonos 0 resolución quirúrgica a través de cirugías como estapedectomía, estapedostomía 0 implante coclear, siendo generalmente considerado como más efectivo el tratamiento quirúrgico. La estapedectomía y estapedostomía son procedimientos bien establecidos en el tratamiento de la otoesclerosis con buenos resultados, generando niveles satisfactorios de audició $\mathrm{n}^{13}$. En nuestra población, si bien se realizaron más estapedostomías que estapedectomías, destaca un número relativamente alto de las últimas, considerando que actualmente en la literatura la primera es la más utilizada ${ }^{14}$. Esto se explica probablemente por el amplio rango temporal de la presente serie, concentrándose en mayor medida las estapedectomías en los pacientes más antiguos. Habitualmente se ha considerado en la literatura como éxito quirúrgico al cierre del gap óseo-aéreo menor 0 igual a $10 \mathrm{~dB} 020 \mathrm{~dB}$, en el $90 \%$ de los pacientes ${ }^{3,4,9-12}$.

Recientemente, en 2017, Karin Strömbäck y Lars Lundman ${ }^{4}$ revisaron las fichas clínicas de 1.688 pacientes para determinar el tipo de técnica quirúrgica utilizada, resultados audiológicos e información demográfica de este grupo de pacientes. La edad promedio fue de 49,5 años, con predominio en el sexo femenino (62\%). La técnica quirúrgica más utilizada fue la estapedostomía $(97,2 \%)$ y la estapedectomía $(2,7 \%)$. Dentro de las complicaciones descritas la de mayor frecuencia fue el tinnitus $(4,7 \%)$, seguido de disgeusia $(2,9 \%)$, vértigo $(1,6 \%)$ e infecciones $(0,7 \%)$. El $90 \%$ de los pacientes reportaron mejoría auditiva al cumplir un año de posoperado. El $63 \%$ de los pacientes presentaron una mejoría mayor a $20 \mathrm{~dB}$ en la vía aérea y el gap óseo-aéreo disminuyó a 10 $\mathrm{dB}$ o menos en el $69 \%$ de los casos.

Por su parte, Alzhrani en 2017 analizó los resultados auditivos de 53 pacientes en los cuales se realizó estapedostomía encontrándose cierre del gap óseo aéreo menor a $10 \mathrm{~dB}$ en el 32,1\% 
de los casos; entre 11-20 dB en el 37,7\%, entre $21-30 \mathrm{~dB}$ en el $22,6 \%$ de los casos. Los autores destacan que es difícil comparar estos resultados con los de otros estudios, ya que algunos calculan la variación el gap óseo-aéreo utilizando la vía ósea preoperatoria para el cálculo del gap óseo-aéreo posoperatorio, lo que ayudaría a mostrar mejores cierres de gap, relacionado con el sobrecierre del gap asociado al efecto de Carhart.

En cuanto a la literatura chilena, Lanas, Stott y Olavarría ${ }^{15}$ analizaron los resultados auditivos de estapedectomía y estapedostomía. En este estudio ambos grupos fueron comparables en cuanto a edad, años de evolución de la enfermedad y distribución por sexo. Todos los pacientes fueron operados por el mismo cirujano. Respecto a los resultados auditivos, en el grupo de las estapedectomías el gap óseo-aéreo del PTP varió de $30 \mathrm{~dB}$ en el preoperatorio a $9 \mathrm{~dB}$ en el posoperatorio $(p$ $<0,05)$, con una mejoría de la vía aérea que pasó de $55 \mathrm{~dB}$ a $30 \mathrm{~dB}$ en el postoperatorio y una vía ósea preoperatoria que, incluso, mostró una leve mejoría $(4 \mathrm{~dB})$ respecto a su valor preperatorio. En el grupo de las estapedostomías se observó algo similar.

En nuestro centro este tipo de cirugía fue efectuada mayoritariamente en mujeres, alcanzando el $67,5 \%$. del total, siendo esto concordante con la mayor proporción de la enfermedad en este género descrita en la literatura ${ }^{15}$. Además, el estado de avance de la enfermedad en el $83,7 \%$ de los pacientes fue bilateral, que también es concordante con otras publicaciones sobre el tema. En nuestro estudio el éxito auditivo definido como cierre del gap óseo-aéreo de $10 \mathrm{~dB} 020 \mathrm{~dB}$ fue de 48,7\% y $76,9 \%$, respectivamente (Tabla 5). Es importante destacar que para calcular el gap óseo-aéreo poso- peratorio se utilizó el umbral óseo posoperatorio.

En la actualidad existe gran heterogeneidad en la forma de reportar los resultados audiométricos. La Academia Americana de Otorrinolaringología y Cirugía de Cabeza y Cuello creó una guía para reportar adecuadamente los resultados audiométricos, buscando unificarlos. Se deben utilizar las frecuencias de 500, 1.000, 2.000 y $3.000 \mathrm{~Hz}$ para calcular el PTP, y se debe especificar el gap óseoaéreo posoperatorio y el cierre de gap (detallando el promedio, desviación estándar y rango), y también la diferencia de umbral auditivo para las frecuencias altas $^{16}$. Luego se agregó el PTP aéreo (promedio, desviación estándar y rango $)^{16}$. Se sugiere especificar todas estas variables para considerar el éxito auditivo. En nuestro estudio los PTP fueron calculados con $4 \mathrm{kHz}$ en lugar de $3 \mathrm{kHz}$. En cuanto a la media de variación de la vía aérea, ésta fue mayor en el grupo de pacientes operados de estapedostomía, sin existir diferencias estadísticamente significativas al realizar test no paramétricos, lo cual se explica probablemente por el número limitado de pacientes en la muestra. En cuanto a las complicaciones, no se encontró asociación entre el tipo de cirugía y una mayor frecuencia de éstas. El porcentaje de reintervención fue de 5,4\% (2 pacientes), siendo esto de menor magnitud que lo descrito en la literatura (20\%-30\%).

\section{CONCLUSIÓN}

La estapedectomía y estapedostomía son procedimientos bien establecidos en el tratamiento de la otoesclerosis con buenos resultados, generando niveles satisfactorios de audición. Las características demográficas y los resultados

Tabla 5. Resultado funcional según distintos criterios de éxito auditivo

\begin{tabular}{|lc|}
\hline Criterio & Éxito auditivo (\%) \\
\hline Gap A- $0 \leq 30 \mathrm{~dB}$ & 87,1 \\
Gap A-0 $\leq 20 \mathrm{~dB}$ & 76,9 \\
Gap A- $0 \leq 10 \mathrm{~dB}$ & 48,7 \\
Delta PTP aére $00 \mathrm{~dB}$ & 91,6 \\
Delta PTP aére $010 \mathrm{~dB}$ & 88,8 \\
\hline
\end{tabular}

A-0: aéreo-óseo 
auditivos obtenidos en nuestro centro, tanto con la estapedectomía parcial y la estapedostomia, son comparables con los descritos en la literatura. No existen diferencias estadísticamente significativas en cuanto al tipo de cirugía y la frecuencia y

\section{BIBLIOGRAFÍA}

1. Czerwinska G, Scierski W, NamyStowski G, Lisowska $G$, MisIoteK M. The surgical treatment results of otosclerosis at the Department of Otolaryngology Silesian Medical University in Zabrze in years 2000-2010. Otolaryngol Pol 2017; 71: 16-21.

2. Thomas JP, Minovi A, Dazert S. Current aspects of etiology, diagnosis and therapy of otosclerosis. Otolaryngol Pol 2011; 65: 162-70.

3. Stott C, OJeda A, Muñoz D, Moyano L. Otoesclerosis: aspectos histopatológicos y resultados auditivos de la estapedostomía. Rev Otorrinolaringol Cir Cabeza Cuello 2012; 72: 125-32.

4. Strömbäck K, Lundman L, BJorsne A, Grendin J, Stjernouist-Desatnik A, Dahlin-Redfors Y. Stapes surgery in Sweden: evaluation of a nationalbased register. Eur Arch Oto-Rhino-Laryngology 2017; 274: 2421-7.

5. Batson L, Rizzolo D. Otosclerosis: An update on diagnosis and treatment. J Am Acad Physician Assist 2017; 30: 17-22.

6. Alzhrani F, Mokhatrish MM, Al-Momani MO, Alshehri H, Hagr A, Garadat SN. Effectiveness of stapedotomy in improving hearing sensitivity for 53 otosclerotic patients: retrospective review. Ann Saudi Med 2017; 37: 499-505.

7. Arias R, Silva M, Veloz M. Estapedostomía endoscópica: experiencia en el Hospital del salvador. Rev Otorrinolaringol Cir Cabeza Cuello 2015; 75: 122-8.

8. Heining C, Banga R, Irving R, Coulson C, Monksfield P. Audiological outcome of stapes surgery for far advanced cochlear otosclerosis. J Laryngol Otol 2017; 131: 961-4. tipo de complicaciones, aunque se observan mejores resultados auditivos en los pacientes operados de estapedostomia. Se requieren más estudios prospectivos para poder definir factores pronósticos de éxito quirúrgico.

9. Husain Q, Lin KF, Selesnick SH. Stapes prosthesis length and hearing outcomes. Laryngoscope 2018; 128: 722-6.

10. Lailach S, Schenke T, Baumann I, Walter H, Praetorius M, Beleites T, et Al. Living with otosclerosis: disease-specific health-related quality-of-life measurement in patients undergoing stapes surgery. Eur Arch Oto-Rhino-Laryngology 2017; 275: 71-9.

11. García-Iza L, Navarro JJ, Goiburu M, Pérez N, Altuna X. Evaluación del cambio en el umbral de la vía ósea en pacientes operados de estapedectomía. Acta Otorrinolaringol Esp 2016; 67: 268-74.

12. Justicz N, Strickland KF, Motamedi KK, Mattox DE. Review of a single surgeon's stapedotomy cases performed with a nickel titanium prosthesis over a 14-year period. Acta Otolaryngol 2017; 137: 442-6.

13. Stott C, Nazar R, Manieu D. Manejo quirúrgico en otoesclerosis unilateral. Rev Otorrinolaringol Cir Cabeza Cuello 2005; 65: 111-16.

14. Délano P, Alvo A, Ojeda A, Stott C. Resultados auditivos y hallazgos quirúrgicos en pacientes con cirugía bilateral por otoesclerosis. Rev Otorrinolaringol Cir Cabeza Cuello 2011; 71: 203-6.

15. Lanas A, Stott C, Olavarría C. Otoesclerosis: Resultados Auditivos de Estapedectomía y Estapedostomía. Rev Otorrinolaringol Cir Cabeza Cuello 2002; 62: 115-22.

16. Committee on Hearing and Equilibrium. Committee on Hearing and Equilibrium guidelines for the evaluation of results of treatment of conductive hearing loss. Otolaryngology-Head and Neck Surgery 1995; 113: 186-7. 